INVESTIGACIÓN

\title{
Cognición contextualizada: Una propuesta didáctica y psicopedagógica socioconstructivista para la enseñanza-aprendizaje del derecho
}

\author{
Contextualized cognition: A didactical and socioconstructivist \\ psycho-pedagógical purposal for teaching-lerning of the Law
}

\author{
Taeli Gómez Francisco y Juan Rubio González \\ Universidad de Atacama, Chile
}

\begin{abstract}
RESUMEN Uno de los fundamentos principales del paradigma educativo socioconstructivista da cuenta de que el proceso de enseñanza-aprendizaje se propicia en la interacción social en comunidades dialógicas y participativas, donde el contexto histórico-cultural del estudiante cumple un factor fundamental. Considerando éste y otros postulados elementales del socioconstructivismo, en el presente trabajo se propone una metodología didáctica y psicopedagógica para la enseñanza-aprendizaje del derecho, orientada a establecer una dialéctica relación entre el aprender y hacer. En esa lógica, se parte describiendo los conceptos que sustentan la base epistemológica del socioconstructivismo y en la que se respalda la propuesta, orientada a implementar un proceso sobre la base de rutas de aprendizaje que, en este caso, constan de seis momentos psicopedagógicos, supeditados al contexto y que, en última instancia, articularán un ciclo didáctico y psicopedagógico de enseñanza-aprendizaje del derecho.
\end{abstract}

PALABRAS CLAVE Socioconstructivismo, enseñanza-aprendizaje del derecho, cognición contextualizada, momentos psicopedagógicos.

ABSTRACT One of the main foundations of the socioconstructivist educational paradigm is that the teaching-learning process is facilitated by social interaction in dialogic and participatory communities, where the student's historical-cultural context fulfills a fundamental role. Considering this and other elementary postulates of socioconstructivism, a didactic and psychopedagogical methodology for the teaching-learning of Law is proposed in this paper, oriented to establish a dialectic relation between learning and doing. In this logic, this paper starts describing the concepts that underpin the epistemological basis of socioconstructivism, and also those that supports the proposal, oriented to implement a process based on «learning paths» that, in this case, consist of six 
psychopedagogical stages conditioned to the context, and that will ultimately articulate a didactic and psycho-pedagogical cycle of teaching-learning of the Law.

KEYWORDS Socioconstructivism, teaching-learning of the Law, contextualized cognition, sychopedagogical stages.

\section{Introducción}

Los paradigmas clásicos, que han sustentado la enseñanza y en particular la del derecho, han desarrollado prácticas educativas en las que explícita e implícitamente se subentiende que el conocimiento puede abstraerse de las situaciones en que se aprende y se emplea, especialmente cuando se trata de leyes que fueron escritas en el siglo XIX, como es el caso de algunos códigos, cuyo objeto de estudio se concibe como un universo pedagógico ideal. En la enseñanza del derecho, éste adopta la característica de un sistema de normas que se autonomizan de quién las dijo, por qué y para qué. Lo que, en términos de paradigmas educativos, ha desembocado en prácticas docentes instruccionales, en las que el aprender y el hacer aparecen como dos dimensiones lejanas y que sólo se encuentran en algunos momentos episódicos. Así, por ejemplo, un estudiante de Derecho puede saber de memoria el proceso penal, razón por la cual califica con excelente nota; sin embargo, no logra desarrollar un argumento jurídico ni una estrategia procesal que requiere para desenvolverse en una situación concreta.

En ello, el docente participa del proceso de manera directiva, ya sea desde un enfoque psicopedagógico conductista, que considera al estudiante como un sujeto pasivo respondiente a diversos estímulos dados, como una ley o cualquier instrucción docente; o desde paradigmas cognitivistas, que si bien plantean un papel más activo al estudiante, de igual manera aíslan el contenido y lo entregan a un depositario que lo debe procesar.

Para ambos paradigmas clásicos, el objeto de estudio se ha concebido como algo dado, y les resulta coherente desarrollar una práctica psicopedagógica, ya sea centrada en una instrucción que premia o castiga con el objetivo de conseguir de parte del estudiante la memorización de lo enseñado, o bien el procesamiento de las mismas. Desde ambos enfoques, se acentúa la enseñanza con un carácter comportamental o cognoscitivo, la que se transforma y convierte en una dualidad en que se produce trasmisión de información por un lado y el aprendizaje por el otro (Fernández, 2005; Sancho-Asensio y otros, 2014; Gómez Mujica y Acosta Rodríguez, 2003).

Esta mecánica de separación de procesos, que prioriza por la información a entregar, tiene como resultante a un estudiante que sólo logra la repetición y reproducción formal de un contenido, ante lo cual desaparece la intencionalidad de la educación como objetivo y como proceso, ya que la trasmisión de información es una actividad 
destinada a rendir «culto a verdades estáticas, acabadas, al almacenaje de cada vez más información, a la memorización y repetición» (Fernández, 2005: 409), e inclusive, pudiéramos agregar, un procesamiento individualizador.

En esta perspectiva dualista, existe una base epistemológica que concibe a un sujeto poseedor de un conocimiento - enseñar- y otro dispuesto a recibirlo - aprender-. Sobresale la figura de un hablante perfecto, abogado-docente-instructor, quien transfiere el conocimiento y, por otro lado, un sujeto-objeto pasivo, dependiente y poco participativo de su proceso de enseñanza-aprendizaje. En esa lógica, la atención está centrada en el producto-verdad. Con ella, se pierde el carácter dialógico y participativo que caracteriza al proceso de enseñanza-aprendizaje y, por cierto, desaparece la noción de una verdad que se construye mediada por la práctica (Pupo, 2014: 110125), por cuanto se posterga la atención de las relaciones humanas, de los sujetos, y se centra en el contenido: en lo que se debe entregar y recibir.

En ese contexto, la atención excesiva al objeto educativo jurídico cosifica las relaciones sociales educativas, lo que genera un fetichismo de los contenidos, con lo cual se invisibiliza la trama compleja del contexto educativo no existiendo una intencionalidad para enriquecerlos, negando las complejidades e incertidumbres, promoviendo la formación de pensamientos simplistas en los estudiantes, con escasa reflexión creativa que permita comprender y participar en las realidades y conflictos, quedando limitados a nivel de teorías, sin proyección heurística para resolver nuevos conflictos jurídicos.

Así, entonces, la no intencionalidad a enriquecer ámbitos educativos dialogantes genera procesos educativos atomizados y compartimentados, que impiden visualizar los objetos de estudio de manera relacionada, como lo son las aulas compartimentadas a una materia, o cohorte, lo que además produce relaciones intersubjetivas poco valoradas, por la atención desmesurada a contenidos - leyes y códigos, en este caso, lo que reduce el aprendizaje que, por el contrario, se vería fortalecido al abordarse desde contextos mediados (Gómez, 2015).

Ante esta situación problemática, la pregunta que surge y que se intenta responder con la propuesta que se dará a conocer en este trabajo, es cómo establecer nuevas bases psicopedagógicas, que permitan dar fundamento al proceso de enseñanzaaprendizaje del derecho - $\mathrm{u}$ otra disciplina - con el objeto de admitir la complejidad tanto del objeto de estudio, como del proceso mismo de relacionarse con él, desde un contexto mediado de relaciones sociales.

Para ello, se propone una metodología que se posiciona en las bases y los principales postulados del socioconstructivismo, con la intención de encontrar en sus propuestas más clásicas los aportes críticos para plantearse contra prácticas psicopedagógicas que centran su proceso de enseñanza-aprendizaje sólo en la transformación de la conducta y la cognición, para, finalmente, enfatizar la importancia de un proceso que considere las relaciones sociales y los contextos prácticos, lo que constituye la 
propuesta de «momentos» didácticos y psicopedagógicos básicos, que permita alcanzar un «aprendizaje contextualizado» del derecho, como un ejemplo paradigmático.

\section{El socioconstructivismo: Paradigma base de la cognición contextualizada}

El paradigma socioconstructivista proporciona una base epistemológica que permite organizar y gestionar la docencia universitaria, como se pretende demostrar. Desde él, se puede acceder a las debilidades e insuficiencias de los paradigmas que centran su atención en la transformación de la conducta o en la cognición sin contexto, y que, de alguna manera, reducen el proceso complejo que caracteriza la formación de un profesional, sea al objeto de estudio - a un cosificado contenido disciplinar reducido a leyes o teorías estancas-, como también a prácticas psicopedagógicas que lo reproducen como el centro del proceso de enseñanza-aprendizaje.

El paradigma socioconstructivista, basado fundamentalmente en los postulados desarrollados en la primera parte del siglo XX por el psicólogo Lev Vigotsky (18961934), considera la enseñanza-aprendizaje como un proceso que se da únicamente en la interacción social. Aprender, entonces, es una actividad social en que los contextos, la comunicación, el lenguaje y las emociones juegan un importante rol, pues se constituyen en herramientas y signos mediadores del proceso de enseñanza-aprendizaje. En ese contexto los sujetos actúan sobre la realidad y en ese proceso la modifican, pero a la vez ellos también se transforman, a través de instrumentos psicológicos denominados «mediadores» (Kozulin, 2000: 22-36; Phan, 2010; Tacca y Branco, 2008). Así entonces, en el fenómeno de la mediación instrumental intervienen herramientas que corresponden a mediadores simples, orientadas hacia los objetos físicos (lápiz, cuaderno, computador, etcétera), y los signos, entendidos como mediadores más sofisticados, que permiten organizar el pensamiento, orientándose hacia el interior y el exterior del sujeto, y produciendo cambios en los otros (lenguaje, creencias, ideas, etcétera).

En ese sentido, para el socioconstructivismo, el lenguaje es una construcción social; Vigotsky (1998: 85-140) plantea al respecto que primero es social, luego es egocéntrico y finalmente es interiorizado, lo cual configura el procesamiento mental (pensamiento), y desde él se genera la cognición humana, la que es expresada a través del mismo lenguaje. Ante ello, el lenguaje se constituye en el principal instrumento mediador, pues les permite a los individuos, en primer lugar, asumirse como seres sociales, porque pueden comunicarse con los demás, exteriorizar sus pensamientos y, además, a través del lenguaje, el ser humano conoce el mundo construyendo sus esquemas mentales, en espacio y temporalidad.

De ahí que el conocimiento, concebido desde el socioconstructivismo, «no es un objeto que se pasa de uno a otro, sino que es algo que se construye por medio de operaciones o habilidades cognoscitivas que se inducen en la interacción social» (Mejía y Sandoval, 1996: 38; véase también Del Caño, 1990; Miras, 1991). En esa perspectiva, 
el conocimiento tampoco sería un algo que el sujeto asimila, como lo plantea el constructivismo clásico de raíz cognitivista-piagetana (Piaget, 1968: 170-173), sino que se produciría un proceso de interiorización-apropiación del conocimiento, pues se provoca la «reconstrucción interna de una operación externa» (Vigotsky, 1987: 92), en la que actúan directamente los contextos socioculturales en que se da el proceso de enseñanza-aprendizaje.

Lo anterior es un elemento clave en el cual detenerse, pues la apropiación está ligada al proceso de interiorización, que corresponde a la fase de transformación de los procesos interpersonales en procesos intrapersonales, pues para el socioconstructivismo las funciones psicológicas aparecen en el sujeto dos veces o en dos niveles: primero a un nivel social y, más tarde, a un nivel individual, vale decir, que se construyen entre personas y después en el interior del individuo (Vigotsky, 1987: 94), de ahí la explicación del origen de que todo cuanto aprendemos está dado por las relaciones entre los seres humanos. En ese sentido, la interiorización implica una reconstrucción del conocimiento, mediante la propia actividad mental de los procesos que ya ocurrieron en el curso de la interacción social. En tanto, la apropiación corresponde al proceso de transformación, al cambio que genera la participación de un individuo en el proceso educativo, pues éste se transforma de tal manera que su intervención en futuros acontecimientos es necesariamente diferente (Vigotsky, 1987: 93-94).

Siguiendo esta explicación, la realidad del estudiante de Derecho, educado desde paradigmas reduccionistas, es la de un sujeto que está aprendiendo en un contexto social reducido, de relaciones sociales de poca interacción, verticales y bancarias —parafraseando a Freire (1985: 77-83) —, salvo esporádicas estrategias didácticas de trabajo cooperativo. Esa realidad, significa en él prácticas reproductoras, sin autonomía, y lenguajes simplificados y con fragilidad de un contenido, que a poco andar puede ser derogado. Por el contrario, de lo que se trata es aprender el objeto de estudio jurídico como una trama mediada por la práctica de un aula de relaciones sociales intencionadas a la interacción de sujetos portadores de conocimientos, ideas, representaciones y valores, en que estudiantes y mediadores construyen, o más bien reconstruyen, nuevo conocimiento a partir de los saberes previos, lo que desde el socioconstructivismo se conoce como «actividad instrumental», que refiere al proceso de formación de las funciones psicológicas superiores, cuestión que ocurre por medio de la actividad práctica e instrumental, no individual, como se postula desde los paradigmas clásicos, sino en la interacción o cooperación social (Frawley, 1999).

En efecto, uno de los argumentos más esclarecedores para hablar de cognición contextualizada está dado por el proceso de formación de las funciones psicológicas superiores, o los llamados «procesos psicológicos superiores», los cuales se caracterizan por ser cualidades específicamente humanas que se desarrollan en los individuos a partir de la incorporación de la cultura, de ahí que su origen sea social (Vigotsky, 1987: 30-45). Esta clasificación se realiza para diferenciarse de los «procesos psicoló- 
gicos elementales», que son comunes al hombre y a otros animales, y se corresponden con la línea natural de desarrollo; ejemplo de ellos son la memoria y la atención que, por lo demás, es el foco de la llamada psicología del aprendizaje, de corte conductista.

Para clarificar lo anterior, el propio Vigotsky relaciona los procesos psicológicos elementales como:

Estructuras primitivas; esto es, un todo psicológico natural, condicionado sobre todo por las peculiaridades biológicas de la psique. A los segundos - los procesos psicológicos superiores, que aparecen en el proceso del desarrollo cultural- los llamaremos estructuras superiores, ya que representan una forma de conducta genéticamente más compleja y superior (Vigotsky, 1987: 129).

En efecto, para el desarrollo concreto de los procesos psicológicos superiores (lengua escrita, atención voluntaria, memoria lógica y emotiva, formación de conceptos, etcétera), no pueden darse descontextualizadamente, separados de la situación o contexto material en que se produce el proceso material de aprendizaje. Al respecto, digamos entonces que un aula jurídica que no promueva la problematización entre pares no puede pretender que el estudiante aprenda a argumentar, a defender sus puntos de vista y situarse desde una posición, si no es una práctica que desarrolle como parte de su formación. En última instancia, es la práctica la que porta el estudiante como aprendizaje, con toda la riqueza relacional que ello implica.

Pero quizás el postulado que establece mayor notoriedad y complejidad a la vez, y que permite establecer una diferencia superlativa entre el socioconstructivismo y otros paradigmas educativos como el conductismo y constructivismo-cognitivista, es comprender el proceso de enseñanza-aprendizaje como actividad contextualizada. Lo anterior hace ruptura con la lógica conductista de entender el aprendizaje como cambio o transformación de la conducta, que tienen en su base el concepto de estímulos y respuestas (E-R), y contradice, además, los postulados del constructivismocognitivista, desde donde se plantea que el aprendizaje implicaría transformaciones en la esfera del pensamiento (cognición), lo que deposita el centro de atención en el estudiante, pero relacionado consigo mismo. En efecto, Edward Lee Thorndike (18741949), uno de los máximos exponentes del conductismo, establece el concepto de conexionismo, como la base del aprendizaje, que vendría como resultado de asociaciones entre estímulo-respuesta, acciones que forman hábitos, que se fortalecen o debilitan, dependiendo de la naturaleza y frecuencia de la relación E-R (Domjan, 2002). En tanto, el constructivismo-cognitivista reconoce el aprendizaje como un proceso único y personal, dado entre el sujeto y el objeto a conocer, en cuyo desarrollo se produciría una construcción individual-interna a partir de esquemas cognitivos previos (Ertmer y Newby, 1993); de ahí que se postula la necesidad de entregar al estudiante, algunas herramientas que le permitan crear sus propios procedimientos, y así poder resolver autónomamente los problemas. 
En ese contexto, el socioconstructivismo supera las categorías de conducta y cognición individual, a través de la categoría de actividad objetivada, que para este paradigma representa la unidad mínima de análisis de la psicología humana (Labra, 1993: 60-64), y la dimensión básica en la cual centrarse para entender el proceso de apropiación del conocimiento. Alekséi Leóntiev (1903-1979) ofrece esta categoría señalando el principio de la relación inseparable entre la psique y la actividad humana, y propone que el proceso de interiorización del conocimiento sucede en la práctica mediante un proceso gradual y dialéctico; la actividad externa va formándose, integral y dialécticamente, en actividad interna (Leóntiev, 1989).

Ante ello, el aprendizaje humano, al ser una actividad objetivada, no puede entenderse como una sumatoria de acciones, sino como una unidad sistémica-compleja y un proceso de construcción social; vale decir, un todo dialécticamente organizado, y no determinado o explicado a partir de las partes que los componen. De ahí que un estudiante no es aquel que reproduce un contenido cuando se le da la instrucción de hacerlo; por el contrario, al estudiante debe reconocérsele como el portador de un proceso, de una práctica. Ésta es la consistencia que le validará su investidura futura como profesional. Desde esa perspectiva, el aprendizaje humano como actividad objetivada no se puede entender como una serie de respuestas a estímulos externos, en los que el estudiante realiza un rol pasivo, influido mecánica y linealmente por el ambiente, pues el sujeto cognoscente opera en una relación social, donde existe una organización compleja y activa, en la cual priman procesos circulares, recursivos y dialecticos, que no sólo transforman al estudiante, sino también al mediador del proceso y, por cierto, a la relación social educativa, donde se da este desarrollo.

En ese contexto, el paradigma socioconstructivista plantea, además, que el proceso de enseñanza-aprendizaje desarrolla una transformación cualitativa, en la llamada «zona de desarrollo próximo», que Vigotsky explica en el contexto de que, en un proceso de enseñanza-aprendizaje, se constatan dos niveles de desarrollo: el actual o presente (nivel real de desarrollo), que expresa lo que el estudiante realmente conoce, determinado por la capacidad de resolver problemáticas de manera independiente; $\mathrm{y}$ el nivel potencial (nivel de desarrollo potencial), que representa lo que el estudiante podría llegar a conocer (Vigotsky, 1979: 133). En ese sentido, surge la zona de desarrollo próximo, que hace referencia a un espacio o nivel de conocimiento que se encuentra entre los niveles reales y potenciales de conocimiento del estudiante. En ese contexto, el socioconstructivismo plantea que, a través de la interacción social y de la actividad mediada, el estudiante construye su conocimiento y puede alcanzar el nivel potencial.

Esta categoría socioconstructivista viene a demostrar que el proceso de aprendizaje es un proceso mediado, en el que se establece una interacción social. En la particularidad, está mediado por sujetos con mayor dominio de alguna temática (que pueden ser docentes o alumnos más aventajados) o también entre iguales (entre pares, 
estudiante-estudiante, docente-docente), quienes ofrecen un andamiaje, entendido como el proceso desarrollado durante la interacción social, en el que un estudiante es guiado o se apoya en otro sujeto para desarrollar su proceso de enseñanza-aprendizaje (Vigotsky, 1979, 130-140).

Desde posiciones reduccionistas y mecanicistas, se plantea que el andamiaje era posible sólo en una relación asimétrica, experto-no experto; sin embargo, el socioconstructivismo desarrolla la lógica simétrica de las interacciones, ante lo cual el andamiaje también puede darse entre iguales (estudiante-estudiante, docente-docente), vale decir, entre aprendices con grados similares de conocimientos, proceso que se conoce como andamiaje colectivo. De lo anterior se desprende que todo proceso de enseñanza-aprendizaje es una acción cooperativa que se da de manera situada o contextualizada, vale decir, existe un contexto material (sociocultural) en el que los participantes exponen y negocian no sólo contenidos, sino historias, culturas, vivencias, emociones y significados.

Desde esa perspectiva, el socioconstructivismo asume que el desarrollo intelectual de una persona está indisolublemente ligado a su medio social y cultural; en última instancia, a su historia ontogenética y a sus patrones culturales. De ahí, la lógica socioconstructivista de entender el desarrollo de los procesos psicológicos superiores, dados primero en el plano social y después en el nivel individual - pues la apropiación de conocimientos y patrones culturales se da en una base de interacción social, vale decir, en un plano interpsicológico-, para luego alcanzar el desarrollo de internalización-apropiación, o sea, en el plano intrapsicológico (Vigotsky, 1979: 93-94). En ese sentido, el socioconstructivismo posiciona al sujeto aprendiz en un contexto históricocultural, y en una situación social del desarrollo determinada, categoría entendida como la «combinación de los procesos internos del desarrollo y de las condiciones internas, que son típicas en cada edad y que condicionan la dinámica del desarrollo psíquico durante el correspondiente período evolutivo» (Bozhovich, 1985: 123; véase también Zaporozhets, 1980), con lo cual se establece que en cada estadio evolutivo, el ser humano presenta una situación de desarrollo particular, definida por el conocimiento adquirido, el desarrollo de sus funciones psíquicas superiores, habilidades, etcétera, que en última instancia representan las herramientas con las que un individuo se enfrenta a un mundo, socialmente determinado.

En esa lógica, el socioconstructivismo plantea que la contextualización de los procesos de enseñanza-aprendizaje deben considerar, además, los procesos afectivos complejos del sujeto, desde donde, en última instancia, surgirá el freno o la estimulación social que permitirán alcanzar o no el conocimiento. En ese contexto, el socioconstructivismo entiende la dimensión social-cognitivo-afectiva, que se da en el proceso psicológico de enseñanza-aprendizaje, como una unidad indisolublemente ligada y en el cual las emociones o el desarrollo afectivo de los sujetos son como el motor que mueve los procesos psicológicos superiores. En ese sentido, las emociones corres- 
ponden a «una tendencia a actuar en una dirección determinada» (Vigotsky, 2004: 40), vale decir, una actividad orientadora del accionar del sujeto, donde se condensan las fuerzas integradoras y motivacionales de la conciencia humana. Así, las emociones cumplen el rol de estructura afectiva, que vinculan simbólica y culturalmente al individuo con la estructura social; de ahí que las personas, al ser moldeadas por las emociones, también determinarán las cualidades de las relaciones sociales, que construyen.

Como parte de los procesos afectivos complejos, que articulan, dinámica e integradamente, el proceso de enseñanza-aprendizaje, el socioconstructivismo también reconoce el rol de las vivencias del sujeto, entendidas como la relación afectiva del individuo con su medio (Fariñas, 2005: 152) y la unidad básica de la situación social del desarrollo como tal, que representa una categoría integrada de elementos externos e internos que se expresan de la misma manera, en aspectos cognitivos y afectivos (González Rey, 2000). Además, el socioconstructivismo le otorga un papel fundamental en el proceso de aprendizaje a las necesidades de los individuos, vistas como elemento rector motivante, que explican la afectividad de los procesos sociales, pues los seres humanos se movilizan por estados de necesidad permanente, de los cuales surgen nuevas necesidades que permiten establecer una contextualización afectiva, en la cual situar un proceso de enseñanza-aprendizaje (González Rey, 200o: 140).

En definitiva, el socioconstructivismo, desde sus clásicas categorías, representa una propuesta coherente para comprender y participar en los procesos educativos actuales. En primer lugar, el sentido de complejidad - que se lo da su base dialéctica- le permite dialogar con los paradigmas de la complejidad que hoy han irrumpido desde los aportes de Morin (2003). Desde el socioconstructivismo, además, se puede sintetizar la relación psique y la actividad humana sin caer en reduccionismos conductuales ni cognitivistas, ampliando la consideración a las necesidades, motivación, a intersubjetividad compleja. Así, la actividad humana representa la relación esencial sujeto-objeto y sujeto-sujeto, en que lo ideal y lo material se convierten recíprocamente, mediante la praxis (Pupo, 2004; Arango-Forero, 2013).

\section{La cognición contextualizada: Una metodología didáctica y psicopedagógica de base socioconstructivista}

Considerando los postulados fundamentales del socioconstructivismo revisados precedentemente, el aprendizaje contextualizado parte de la premisa fundamental de que el conocimiento es una construcción social. Por lo tanto, es social histórica y culturalmente situado, vale decir, es parte y producto de la actividad que desarrollan los individuos interactuantes, del contexto y de la cultura en que se desarrolla y utiliza (Díaz Barriga Arceo, 2003; Reyes García, Vera Guadrón y Colina Caldera, 2014). Desde esa perspectiva, el conocimiento no puede abstraerse de las situaciones en que 
se aprende y se emplea. No sucede de igual manera con la práctica psicopedagógica declarativa, abstracta y descontextualizada, que genera algunas propuestas educativas basadas en enfoques conductistas y cognitivistas del aprendizaje, de lo que resultan conocimientos independientes de las prácticas sociales o la vida real de las personas, todo dado por una pretendida neutralidad que se le intenta adjudicar al conocimiento, lo que causa una evidente ruptura entre el qué saber y el cómo saber (Díaz Barriga Arceo, 2003: 110; Reyes García, Vera Guadrón y Colina Caldera, 2014).

Frecuentemente, se responsabiliza a los estudiantes por su incapacidad de aplicar contenidos, transferir o generalizar lo aprendido, como también a los docentes se les critica por su falta de didácticas para transmitir los contenidos. Esa lógica focaliza la problemática en las partes, sea en el estudiante o docente; sin embargo, desde el socioconstructivismo se considera relevante el ámbito de la relación social: en última instancia, es en el contexto social donde los sujetos aprenden, tanto estudiantes, como docentes. A fin de cuentas, se plantea como un proceso de socialización y enculturación, ante lo cual, el aprender y hacer son acciones sociales dialécticamente inseparables, y además procesos multidimensionales (involucran cognición, afectividad y práctica concreta). A diferencia de un aprendizaje descontextualizado, que hace la ruptura antes señalada (qué saber-cómo saber).

En ese marco, la cognición contextualizada, como parte del paradigma socioconstructivista, asume que el proceso de enseñanza-aprendizaje implica, como primer apronte, el entender e internalizar los símbolos y signos de la cultura y grupo social al que se pertenece; es en ese proceso, mediado por la interacción social, que el estudiante se apropia de prácticas y herramientas culturales que constituyen su base intelectual (saberes previos), con las cuales se negociarán los nuevos significados y conocimientos. De la comprensión de estos aspectos básicos dependerá, entonces, el resultado de los siguientes procesos de interacción, de andamiaje entre mediadorestudiante y entre éste y sus pares.

De lo anterior se desprende que el aprendizaje contextualizado requiere de estrategias y didácticas psicoeducativas que promuevan la reflexión en la acción, que sean adaptativas y extrapolables, direccionadas a situaciones específicas y reales, buscando resolver problemas surgidos de situaciones cotidianas. Para lograrlo, se incentiva el trabajo cooperativo, en equipo o metodologías que conlleven la necesaria participación, relaciones y vinculaciones de los protagonistas del proceso de enseñanzaaprendizaje, pues se entiende que éste únicamente se desarrolla en un contexto social. Aprender es una experiencia social, que se potenciará con las experiencias y vivencias de otros, las que, asimismo, se fortalecen con la experiencia del aprendiz.

Como se planteó, la participación de docentes, sujetos más experimentados y pares más aventajados, serán factores mediacionales del proceso, que no desarrollan sólo prácticas instruccionales, sino más bien se transforman en agentes de enseñanza; sujetos que estratégicamente deben intencionar el proceso de enseñanza-aprendizaje, 
configurando contextos reales, espacios motivacionales y generando estrategias psicoeducativas, más que centrarse en un contenido o materia en específico. En términos prácticos, el agente de enseñanza debería configurar un colectivo psicopedagógico y metodológico, basado en el dialogo y la participación como factores fundamentales en la construcción de conocimiento. De ahí que no sólo las aulas son espacios de aprendizaje, sino todo contexto o entorno donde exista interacción social. El agente de enseñanza, en suma, debe propiciar claramente la unión, sinergia y complementariedad, entre el qué saber y el cómo saber (Díaz Barriga Arceo, 2003: 115).

\section{Algunas estrategias pertinentes a utilizar en el aprendizaje contextualizado}

La intención de este trabajo no es en centrarse en las estrategias o procedimientos desarrollados a partir del aprendizaje situado, que la literatura considera y trabaja en profundidad (Díaz Barriga Arceo, 2003: 108). Sin embargo, parece pertinente considerar y explicar, a grandes rasgos, algunas experiencias de estrategias transversales, desarrolladas a partir de esta lógica, en las que se establece claramente que el propósito del aprendizaje no puede entenderse como un fin en sí mismo, sino que se debe dar relevancia a la actividad social y a la interacción entre las personas, pues se destaca la dimensión social de los procesos en la adquisición de competencias de los individuos.

En ese sentido, como estrategias transversales que contribuyen a desarrollar el aprendizaje contextualizado en un estudiante de Derecho, se destacan:

- La solución de problemas auténticos, que consiste en presentar al estudiante situaciones reales que deben vincular en ejercicios guiados, con un contenido determinado y asociado a un ámbito de conocimiento o ejercicio profesional. Se busca, básicamente, que el estudiante pueda analizar la situación, tomar posición, problematizar y construir una o varias propuestas viables de solución. En ese ejercicio problémico de aplicación e integración, el estudiante debería retener, profundizar y comprender de mejor manera los contenidos entregados. Una variante similar a la expuesta es la estrategia de aprendizaje, utilizada mediante el análisis y resolución de casos, o la simulación o juegos, mayormente utilizada en ámbitos escolares básicos (Díaz Barriga Arceo, 2003: 112).

- La estrategia de aprendizaje mediante proyectos (Díaz Barriga Arceo, 2003: 112), en que se asigna a un grupo pequeño de estudiantes un ejercicio formal que les permita desarrollar actividades, que involucre investigar, construir y analizar información, pero focalizaoa al objetivo específico de la temática planteada. La idea básica es que el estudiante genere capacidad para desarrollar preguntas clave, direccionada a problemáticas particulares que deben ser resueltos, mientras de paso desarrollan capacidad de síntesis, pensamiento crítico-complejo, autonomía y responsabilidad, pues deberán planificar y estructurar su propia 
metodología investigativa, en lo posible con retroalimentación colectiva, no como un acto posterior, sino integrante de la estrategia planificada.

- La investigación bibliográfica (Crowther, 1993; Eberhardt y Heinz, 2017; Ruiz Hoyos y Cardona Rivas, 2016), que consiste en instruir al estudiante para que revise material impreso o en la web (libros, periódicos, revistas científicas, tesis, monografías, etcétera) que se relacione con la temática trabajada, de manera tal que el sujeto aprenda a identificar y seleccionar información precisa y atingente a los temas investigados. De paso, esta estrategia permitirá acercar al estudiante a desarrollar una lógica formal en la construcción de productos investigativos, establecer referencias y, sobre todo, comenzar a hacer dialogar diversos conocimientos y posturas sobre una temática en particular. Con ella, se trae a los autores y la red bibliográfica al aula para, con esta variedad de sujetos y autores, interactuar.

- Estrategias de aprendizaje, basadas en escenarios reales (in situ), buscan que el estudiante se desarrolle y practique el conocimiento mediante la participación activa en espacios comunitarios (Wenger, 2001; Figueira Santos Pereira, 2016; Godói-de-Sousa y Nakata, 2013), lo que se conoce como «actividades de servicio a la comunidad» (service learning), en las que el sujeto cognoscente puede aplicar los conocimientos y las habilidades adquiridas en situaciones de la vida real y en sus propias comunidades, con lo cual se extiende el proceso de enseñanza-aprendizaje más allá del aula, además de fomentar la lógica de sentido de responsabilidad y empatía social, no con un carácter caritativo, sino desde una lógica «relacionada con una toma de conciencia moral, social y cívica» (Díaz Barriga Arceo, 2003: 113).

- Otras estrategias de aprendizaje, relacionadas al trabajo en equipos cooperativos (Johnson, Johnson y Holubec, 1999; Fernández-Rio y otros, 2017; León del Barco y otros, 2017) que, a diferencia de las lógicas individualistas y competitivas, busca que el estudiante alcance su objetivo propuesto en la medida en que sus compañeros de equipo o su grupo alcancen sus objetivos. Lo que se persigue con estos procedimientos es atribuir el éxito de un plan o meta tanto al esfuerzo personal (motivación interna) como al esfuerzo colectivo, lo cual establece el convencimiento futuro de que todos los integrantes de un equipo tienen algo que aportar e influir en los demás, y que la interacción con los compañeros será fundamental para elaborar y construir conocimiento.

En general, estas propuestas de estrategias transversales son favorables para considerar la relevancia del contexto social y de la interacción intersubjetiva y de teoríapráctica. Sin embargo, con todo el favorecimiento como técnicas particulares, este 
trabajo se propone superar estrategias individuales y plantear una metodología didáctica y psicopedagógica de aprendizaje contextualizado del derecho.

\section{Metodología didáctica y psicopedagógica de aprendizaje contextualizado del derecho}

De acuerdo a lo revisado hasta el momento, un aprendizaje contextualizado sólo se da si se está frente a un proceso dinámico, activo y centrado en experiencias motivantes para los protagonistas. De la misma manera, requiere propiciar espacios o atmosferas dialogantes, de trabajo cooperativo y discusión grupal que permita a los agentes de aprendizaje mediar y direccionar el proceso de enseñanza-aprendizaje. Desde esa perspectiva, se propone orientar un desarrollo metodológico didáctico y psicopedagógico que permita establecer un aula basada en el paradigma socioconstructivista, donde converjan el contexto sociocultural, la capacidad mediadora del agente de enseñanza y las habilidades del estudiante, para lo cual se establecen ciertos momentos psicopedagógicos que se deben mediar no lineal o mecánicamente, sino de manera sistémico-compleja y en directa relación o determinación del contexto del aula.

Cabe señalar que la propuesta que se realiza se relaciona en la forma, en alguna medida, con los momentos de aprendizaje desarrollados por Coll (Coll, 1991; CruzGarcette, De Abreu-Ballester y Brandi-Bruna, 2014; Gutiérrez-Giraldo y López-Isaza, 2011), desde un enfoque cognitivo, también con las didácticas de aprendizaje significativo de Ausubel, Novak y Hanesian (Ausubel, Novak y Hanesian, 1995; Valderramas Gomes, 2014; Gonçalves y otros, 2014), pero básicamente se diferencia porque, en este caso, se orienta un desarrollo teórico-práctico desde el paradigma socioconstructivista; de ahí que se asemeje más a una adecuación de las rutas de aprendizaje propuestas por Pacheco y Porras (Pacheco Peña y Porras Cosme, 2014; Jerónimo, Sepúlveda Solá y Bonet Garrosa, 2007), entre otros.

En ese sentido, se plantea un proceso a implementar en el aula (o fuera de ella) en base a seguir rutas de aprendizaje, vale decir, establecer un tránsito o una secuencia determinada por el contexto, por las particularidades de la comunidad de aprendizaje. De ahí que no se puede proponer una ruta a priori, sino que ésta debe ir surgiendo desde la práctica misma. Este tránsito consta de seis momentos psicopedagógicos básicos, que corresponden a los diversos hitos, tareas o pasos, que debe seguir la ruta de aprendizaje propuesta, y que, por cierto, obedecerán a la lógica de poder configurar una comunidad de aprendizaje dialógica y permanente, que posibilite un aprendizaje sistemático y contextualizado.

Lo importante a resaltar en este caso es que estos momentos psicopedagógicos no corresponden a una práctica lineal, ni mecánica, sino se deben supeditar al contexto, ya que, en última instancia, articularán un ciclo didáctico y psicopedagógico, cuya temporalidad debe ser definida desde el contexto mismo. En la práctica, el ciclo dará 
cuenta de la realización de la mayor parte de los momentos psicopedagógicos en un espacio temporal, que deberá ser definido desde el contexto mismo y que, por cierto, traspasa los espacios temporales y espaciales de las clases o cátedras convencionales. Así, un ciclo didáctico y psicopedagógico puede estructurarse en una o varias clases, actividades, talleres e incluso integración de asignaturas y, sin duda, utilizando diversos medios y didácticas, espacios y tiempos.

Antes de detallar el ciclo didáctico y psicopedagógico y los momentos psicopedagógicos en particular, es conveniente aclarar que se parte de la base de que la unidad académica donde se implemente la propuesta cuenta con un soporte psicopedagógico básico de apoyo al grupo y al agente de aprendizaje. En relación a este último, se considera también que el docente mediador del proceso conoce y tiene dominio de las categorías y metodologías socioconstructivistas, relaciona saberes diversos que le permiten desarrollar en el aula, conocimientos, habilidades y herramientas cognitivas, capacidades interpersonales, etcétera. Además, maneja a cabalidad el contenido que desarrollará en sus clases.

Considerado lo anterior, se detallan los momentos didácticos y psicopedagógicos básicos para alcanzar un aprendizaje contextualizado del derecho:

\section{Mapeo de las representaciones sociales del estudiante}

Esta fase previa a la práctica en el aula parte de la lógica de que no se puede desarrollar un proceso de enseñanza-aprendizaje sin conocer las necesidades e intereses de los estudiantes y de su entorno colectivo-social. De ahí que esta fase se presenta como ineludible y sustancial para desarrollar el proceso, pues el agente de enseñanza debe reconocer en términos generales la realidad social y cultural del grupo de aprendices, vale decir, cantidad de estudiantes en aula, estar al tanto de las condiciones económicas y de procedencia, rasgos etarios y experiencias previas.

En lo posible, la institución debe propiciar la caracterización de sus estudiantes, al estilo de lo realizado por la Unidad de Pedagogía Universitaria y Didáctica del Derecho de la Universidad de Chile (Elgueta, Zamorano y Palma, 2015). En este sentido, es necesario señalar que en la actualidad se han ido creando en diversas instituciones unidades de apoyo a la docencia, las que facilitan el conocimiento de elementos básicos respecto la cognición del colectivo de aprendizaje, como estilos y ritmos de aprendizaje, canales perceptivos que prevalecen en el grupo e inclusive información relevante proveniente de las nuevas orientaciones de la universidad inclusiva.

Así entonces, esta fase de mapeo debe realizarse en conjunto con una red de apoyo psicopedagógica que le entregue la información necesaria al agente de aprendizaje y le proporcione el soporte metodológico para su práctica en el aula. Cabe señalar que esta fase, a medida que el agente de enseñanza se vaya relacionando y profundice la 
relación con el colectivo de aprendizaje, irá obteniendo mayor información, la que nutrirá el mapeo de las representaciones sociales del grupo de aprendizaje.

De lo anterior se desprende que, metodológicamente, en este momento psicopedagógico se parte de lo general a lo particular, considerando además que debería ir acompañada de una evaluación diagnóstica al grupo de aprendizaje y de la recopilación del máximo de antecedentes, lo que permita al agente de aprendizaje operar con información objetiva y confiable. En ese sentido, es básico el dialogo con los demás docentes, agentes de enseñanza y otros actores relevantes, quien permitirán conocer experiencias anteriores y estrategias de enseñanza-aprendizaje utilizadas. Al mismo tiempo, se pueden instalar ciertas prácticas educativas que propicien un acercamiento de todos, como presentaciones, la creación de un convenio de expectativas colectivas o compromisos, etcétera.

\section{Motivación inicial y encuadre del grupo de aprendizaje}

En esta fase del proceso, el agente de enseñanza establece el rapport, vale decir, crea y organiza el ambiente o clima del colectivo de aprendizaje. Se posiciona al estudiante en situación de aprendizaje, persuadiendo y provocando su curiosidad e interés, y, sobre todo, se moviliza su estructura afectiva; se motiva a partir del surgimiento de un contexto de confianza, familiaridad y acogida en el aula.

$\mathrm{Si}$ bien este momento psicopedagógico pudiese entenderse como transversal a todo el ciclo, se sugiere que exista una fase de encuadre, en la que se establezca el marco, objetivos y secuencias del ciclo didáctico y psicopedagógico, y, a la vez, se produzca la activación de los conocimientos previos del estudiante, entendido no sólo como el considerar los saberes factuales y conceptuales, centrados en una materia o contenido determinado que se proponen desde el paradigma cognitivista, sino también activar las herramientas actitudinales y procedimentales del estudiante, vale decir, lo que Vidal-Abarca y Martínez señalan como los esquemas de interpretación o constructos psicológicos (Vidal-Abarca y Martínez, 1998; véase también Neira Martínez, Reyes Reyes y Riffo Ocares, 2015; Guerra García y Guevara Benítez, 2017; Fajardo, Hernández y González, 2012), que se encuentran en la base de todo tipo percepcióninterpretación de información, donde las necesidades, vivencias, creencias, concepciones y emociones presentes en el estudiante son factores decisivos a considerar al momento de enfrentarse con un nuevo conocimiento.

En tal contexto, la lógica en esta fase es activar las capacidades cognitivas, junto con las estructuras mentales y socioculturales del estudiante, pero sobre todo movilizar y persuadir los procesos afectivos complejos que pueden ser mediados a través de «experiencias lúdicas, ecológicas o culturales de su medio social que capten la atención inicial del estudiante» (Pacheco Peña y Porras Cosme, 2014: 80). Así entonces, la motivación inicial y encuadre buscan desatar el conflicto actitud-predisposición en 
el colectivo de aprendizaje, estimulando la voluntad y despertando el razonamiento, pero también estableciendo un marco adecuado a las expectativas que se pudiesen generar, entendido ello como el implícito y explicito arribo de consenso, sobre un marco contextuado, que dará base y sustento al proceso de enseñanza-aprendizaje.

\section{Momento del conflicto cognitivo y problematización}

En esta fase del proceso metodológico, el agente de aprendizaje expone los nuevos contenidos en contexto utilizando didácticas atrayentes y de fácil manejo para el estudiante, establece conceptos, ideas fuerza, orientaciones diversas sobre la temática, su propia opinión, etcétera, tras lo cual se debería propiciar la reflexión de saberes en el colectivo de aprendizaje a partir de los conocimientos previos y los nuevos saberes, vale decir, se problematiza el pensamiento y saber del estudiante, se produce el conflicto cognitivo entre lo nuevo y lo previo, se propicia la polémica situada en torno al contenido planteado y, por cierto, la problematización instala la incertidumbre, el dilema y la incerteza en el colectivo de aprendizaje. La idea, en esta lógica, es que el estudiante se enfrente con una diversidad de respuestas ante el desequilibrio cognitivo planteado que le pueden generar el enfrentamiento de saberes nuevos y previos, expectativas, creencias, etcétera.

Atendiendo la lógica socioconstructivista de que es el lenguaje social el precursor del pensamiento hablado y de todas las variantes cognitivas, en esta fase se sugiere promover la mayor diversidad de procesos de comunicación y participación compartida en actividades, pues ello permitirá comprometer al estudiante en la tarea de ampliar su conocimiento y su posterior aplicación a nuevas problemáticas o situaciones. Desde esa perspectiva, en esta fase del proceso se produciría una apertura y activación de los procesos psicológicos superiores, vale decir, se pasa de la prehistoria del aprendizaje, centrado sólo en la atención y memoria de un contenido, a una fase de internalizaciónapropiación, en que la interacción social y el andamiaje del proceso, cumplen un rol esencial, pues de las interrogantes aquí planteadas surgirá la posterior reflexión contextualizada, en que el conflicto cognitivo y la problematización ponen a prueba las configuraciones del estudiante y la capacidad mediadora del agente de aprendizaje.

En este momento psicopedagógico se sugiere realizar un tránsito desde el dialogo docente-estudiante al de estudiante-estudiante, pues lo que se requiere propiciar es el trabajo en equipo, que promueve el surgimiento de diversas ideas e hipótesis y desemboque en un razonamiento cooperativo.

\section{Momento del conflicto práctico y resignificación}

La lógica en esta fase es accionar el cerebro emotivo (Ledoux, 1999; Vera-Villarroel y otros, 2010) para que los simbolismos se conecten con los elementos objetivos pre- 
sentes en el contenido, con lo que se desarrollan actividades prácticas que permitan producir el nexo y el tránsito entre la memoria a corto plazo y la memoria de largo plazo a través de la práctica dialógica, además de acciones que permitan comprender, sistematizar y consolidar el conocimiento.

En la práctica concreta, esta fase del proceso no corresponde sólo a instalar didácticas de reforzamiento de contenido, sino también a generar un andamiaje psicopedagógico que propicie un conflicto práctico, vale decir, poner en jaque las habilidades del estudiante en relación con situarse en el contexto y no el contenido: el contenido no es un algo que se debe aprender o memorizar, sino un objeto con el cual el estudiante debe relacionarse, pero desde un contexto situado (Gómez, 2015). Lo anterior implica relacionar lo simbólico con lo práctico, evidenciando que los significados que se le dan a un contenido determinado dependen del contexto; es el medio el que carga de significado al contenido, lo que lleva a romper con las categorías estancas por un lado y establecer, por otro, que existen prácticas comunes que orientan ciertos tipos de significados de un contenido.

En ese sentido, este momento psicopedagógico propuesto busca la resignificación del estudiante respecto de la relación objetual con el contenido, entendido dogmáticamente como el que rige el aprendizaje, pero que desde una lógica contextualizada no es más que un objeto relacional que en la práctica debe ser conceptuado desde el contexto, lo que significa darle un sentido diferente: una reubicación o reorientación al nuevo saber a la práctica previa, a partir de una nueva comprensión del conocimiento.

\section{Momento de evaluación, metacognición y reforzamiento}

Es la fase del ciclo cuando la comunidad de aprendizaje se autoevalúa, con lo que genera el conflicto de consolidación-destrezas adquiridas, frente a las actitudes y motivaciones que presenta el estudiante. Se desarrolla la metacognición, vale decir «un monitoreo mental» (Pacheco Peña y Porras Cosme, 2014: 82) en que se ponen a prueba las estrategias utilizadas para apoderarse del conocimiento, pero también el convencimiento del estudiante, reflejado en el tipo de reflexión que realiza.

Idealmente, la evaluación, metacognición y reforzamiento debería ser una fase transversal al ciclo didáctico y psicopedagógico, abordada no de manera sumativa, sino sistémicamente, pues la práctica dialógica permitiría ir desarrollando el proceso dialécticamente; los agentes de enseñanza despliegan su rol mediador, de andamiaje y guía, durante el cual el docente puede apoyarse en individuos más aventajados de la comunidad de aprendizaje para desarrollar e intencionar el proceso. Este momento Psicopedagógico se debe aprovechar para realizar alfabetización emocional, con lo que refuerza autoestima y autonomía, entrega oportunidades para la reflexión propia y establece direccionalidad respecto al comportamiento ético y valorativo del estudiante. 
Se sugiere que esta fase sea además transversal, pues a partir de ella el agente de aprendizaje evaluará la consolidación del aprendizaje en el estudiante, valorará sus capacidades, habilidades, destrezas, conocimientos, actitudes y valores; por cierto, también obtendrá la información necesaria para adecuar y orientar el proceso de enseñanza-aprendizaje. Desde esa perspectiva, durante la práctica deberían desprenderse indicadores de refuerzo del proceso, que el agente de enseñanza necesita considerar para establecer mediciones, controles y adecuaciones.

En esa aproximación de la reflexión colectiva, que dé cuenta del proceso en general, de los procedimientos utilizados, de las dificultades, de las medidas correctivas, de la conceptualización y de la actitud del estudiante, el agente de enseñanza debe hacerse cargo sistematizando el proceso, ahondando en lo esencial del contenido tratado, resaltando los puntos vulnerables que apreció y realizando la retroalimentación, tras lo cual la comunidad de aprendizaje puede contrastar lo aprendido.

\section{Momento de extensión, integración y de dar sentido al conocimiento}

Se arriba a un momento psicopedagógico en que la comunidad de aprendizaje ha integrado el conocimiento, de tal manera que éste se hace dinámico y extensible hacia otras prácticas o áreas de aprendizaje. Es el momento cuando el saber adquiere sentido; se genera el conflicto de adquirir conciencia del saber aprendido frente a la relación con la práctica concreta de este conocimiento, vale decir, la comunidad de aprendizaje hace parte suya el saber (apropiación-interiorización), lo cual le permite posicionarlo en la realidad y, por cierto, darle proyección heurística.

En términos prácticos, el estudiante en esta fase está en condiciones de relacionar el conocimiento y principalmente reconocer la funcionalidad del mismo, cómo el saber impacta en las diversas áreas o disciplinas, que en última instancia sería lo que se podría evaluar - trasladando ello a una lógica de evaluación directiva一; en fin, estableciendo la relación y funcionalidad de un contenido determinado se podría aventurar a saber, si el estudiante realmente sabe, si está en condiciones de darle utilidad al conocimiento y, por cierto, reconocerlo, discriminarlo y hacerlo conversar con la práctica concreta.

\section{Conclusiones}

La propuesta metodológica dada a conocer no se plantea de manera neutral ni ecléctica, pues se posiciona desde un paradigma cuyo basamento epistemológico se ha dado a conocer en Occidente de manera desmembrada y descontextualizada, de ahí que muchas veces aparecen ciertas prácticas como contradictorias. Efectivamente, el socioconstructivismo parte de premisas que no se pueden obviar en la práctica psicopedagógica: principalmente, entiende que todo proceso psicológico es un proceso 
social. Vale decir, que el aprendizaje se da únicamente en un marco de interacción social y, por sobre todo, que considera las condiciones concretas de la existencia de los individuos.

De ahí la necesidad de considerar que un proceso de enseñanza-aprendizaje debe plantearse desde una lógica contextualizada, dinámica y adaptativa, características que, consideramos, contiene la propuesta metodológica realizada, en la que no se establece linealidad ni sugiere tiempos o plazos, pues se entiende que el contexto irá condicionando el proceso práctico. Por ello, se plantea en términos de considerar una ruta de aprendizaje conformada por ciertos momentos psicopedagógicos que conformen un ciclo didáctico y psicopedagógico, que configuren una comunidad de enseñanza-aprendizaje dialógica y permanente.

En ese contexto, desde una perspectiva dialéctica, la propuesta considera que todo proceso se configura a partir de contradicciones, de nudos teórico-prácticos que se deben enfrentar y resolver para avanzar. En ese sentido, se hace cargo de un sistema educacional en que la incertidumbre y el caos son cualidades emergentes a considerar.

De la misma manera, la propuesta se aborda con una perspectiva de intervención peda-psicoterapéutica, pues se asume que todo proceso de enseñanza-aprendizaje debe partir desde un continuo permanente que establece el reconocimiento del contexto, pasando por una fase de problematización en la que se intenta desnaturalizar lo ya naturalizado por la práctica sociocultural, lo cual lleva a una resignificación del saber, que es la fase necesaria para luego alcanzar la concientización o etapa en que el contenido adquiere sentido. Vale decir, el saber se puede relacionar y darle funcionalidad estratégica.

\section{Referencias}

Arango-Forero, Germán (2013). «Comunicación digital: Una propuesta de análisis desde el pensamiento complejo». Palabra Clave, 16 (3): 673-697. DOI: 10.5294/ pacla.2013.16.3.1.

Ausubel, David, Joseph Novak y Helen Hanesian (1995). Psicología educativa: Un punto de vista cognoscitivo. Ciudad de México: Trillas.

BozHovich, Lidia Ilinichna (1985). La personalidad y su formación en la edad infantil. La Habana: Pueblo y Educación.

Coll, César (1991). Aprendizaje escolar y construcción del conocimiento. Barcelona: Paidós.

Crowther, Warren (1993). Manual de investigación-acción para la evaluación en el ámbito administrativo. San José: EUNED.

Cruz-Garcette, Lorena, Graciela Martins de Abreu-Ballester y Lía Stella BRANDI-BRUNA (2014). «La relación intersubjetiva en la construcción del conocimiento». Magis, 7 (14): 85-98. DOI: 10.11144/Javeriana.M7-14.LRIC. 
DelCAÑo,Maximiliano(1990).«Interacción entreiguales, mediosocialy desarrollocognitivo».Infancia y Aprendizaje, 13 (50):27-42.DOI:10.1080/02103702.1990.10822264.

Domjan, Michael (2002). Bases del aprendizaje y el condicionamiento. $2 .^{a}$ ed. Jaén: El Lunar.

Díaz BARriga Arceo, Frida (2003). «Cognición situada y estrategias para el aprendizaje significativo». Revista Electrónica de Investigación Educativa, 5 (2): 105-117. Disponible en http://bit.ly/2jsc6Ct.

Eberhardt, Annelie y Manuela Heinz (2017). «Walk little, look lots: Tuning into teachers action research rhythm». Studying Teacher Education, 13 (1): 36-51. DOI: 10.1080/17425964.2017.1286578.

Elgueta, María Francisca, Francisco Zamorano y Eric Palma (2015). Primer estudio nacional de caracterización de estudiantes de Derecho. Santiago: Unidad de Pedagogía Universitaria y Didáctica del Derecho, Facultad de Derecho, Universidad de Chile.

ErTmer, Peggy y Timothy Newby (1993). «Behaviorism, cognitivism, constructivism: Comparing critical features from an instructional design perspective». Performance Improvement Quarterly, 6 (4): 50-72. DOI: 10.1111/j.1937-8327.1993.tboo605.x.

FajArdo, Adriana, Janeth Hernández y Ángela GonzÁlez (2012). «Acceso léxico y comprensión lectora: Un estudio con jóvenes universitarios». Revista Electrónica de Investigación Educativa, 14 (2): 25-33. Disponible en http://bit.ly/2jrPwtY.

FARIÑAs, Gloria (2005). Psicología, educación y sociedad: Un estudio sobre el desarrollo humano. La Habana: Félix Varela.

FERNÁNDEZ, Lourdes (2005). «Trabajar en grupos en los contextos educativos». En Martha Alejandro y María Isabel Romero (compiladoras), Trabajo grupal y coordinación: Selección de lecturas (pp. 405-414). La Habana: Caminos.

Fernández-Rio, Javier, José Cecchini, Antonio MéndeZ-Giménez, David MénDEZ-Alonso y José Prieto (2017). «Design and validation of a questionnaire to assess cooperative learning in educational contexts». Anales de Psicología, 33 (3): 680-688. DOI: 10.6018/analesps.33.3.251321.

Figueira Santos Pereira, Maria da Conceição (2016). «Comunidades de práctica y desarrollo profesional docente». Opción, 32: 736-747. Disponible en http://bit. ly/2jrdRQz.

FraWley, William (1999). Vigotsky y la ciencia cognitiva. Barcelona: Paidós.

Freire, Paulo (1985). Pedagogía del oprimido. Ciudad de México: Siglo XXI.

Godói-DE-Sousa, Edileusa y Lina Eiko NAKATA (2013). «Comunidades de práctica. Una innovación en la gestión del conocimiento». Journal of Technology Management and Innovation, 8 (1): 124-134. DOI: 10.4067/So718-27242013000300011.

Gómez, Taeli (2015). «Andamiar hacia las incertidumbres: Una propuesta educativajurídica». Participación en Congreso del Instituto de Investigaciones Jurídicas de la UNAM, Ciudad de México. 
Gómez Mujica, Aleida y Heriberto Acosta Rodríguez (2003). «Acerca del trabajo en grupos o equipos». ACIMED, 11 (6): 1-21. Disponible en http://bit.ly/2AB8Tdr.

GonzÁlez Rey, Fernando (2000). «El lugar de las emociones en la constitución social de lo psíquico: El aporte de Vigotsky». Educação \& Sociedade, 21 (70): 132-148. DOI: 10.1590/So101-73302000000200006.

Gonçalves, Óscar, Adriana Sampaio, Ana Raquel Mesquita, Ana Osório y Patricia Oliveira-Silva (2014). «A psicologia como neurociência cognitiva: Implicações para a compreensão dos processos básicos e suas aplicações». Análise Psicológioca, 32 (1): 3-25. DOI: 10.14417/ap.836.

Guerra García, Jorge y Carmen Yolanda Guevara Benítez (2017). «Variables académicas, comprensión lectora, estrategias y motivación en estudiantes universitarios». Revista Electrónica de Investigación Educativa, 19 (2): 78-90. DOI: 10.24320/ redie.2017.19.2.1125.

GutiérRez-Giraldo, Martha Cecilia y Giovanni López-Isaza (2011). «La interactividad en la construcción del conocimiento escolar». Revista Innovar, 21 (41): 5-17. Disponible en http://bit.ly/2ABYTR3.

Jerónimo, Inmaculada, Jara Sepúlveda Solá y Marta Bonet Garrosa (2007). «Estandarización de rutas didácticas para compartir el aprendizaje: Una propuesta real de desagregación y secuenciación de objetos». CEUR Workshop Proceedings 318. Disponible en: http://bit.ly/2ADFwXV.

Johnson, David, Roger Johnson y Edythe Holubec (1999). El aprendizaje cooperativo en el aula. Buenos Aires: Paidós.

Kozulin, Alex (2000). Instrumentos psicológicos: La educación desde una perspectiva sociocultural. Barcelona: Paidós.

LABRA, Iván (1993). Psicología social, responsabilidad y necesidad. Santiago: Lom.

LEDoux, Joseph (1999). El cerebro emocional. Barcelona: Ariel-Planeta.

León del Barco, Benito, Santiago Mendo-Lázaro, Elena Felipe-Castaño, MaríaIsabel Polo del Río y Fernando FajARdo-Bullón (2017). «Team potency and cooperative learning in the university setting». Revista de Psicodidáctica, 22 (1): 9-15. DOI: 10.1387/RevPsicodidact.14213.

-. (1989). La actividad en la psicología. La Habana: Pueblo y Educación.

MejíA, Rebeca y Sergio Sandoval (1996). Interacción social y activación del pensamiento. Guadalajara: ITESO.

Miras, Mariana (1991). «Educación y desarrollo». Infancia y Aprendizaje, 14 (54): 3-17. DOI: $10.1080 / 02103702.1991 .10822290$.

Morin, Edgar (2003). Introducción al pensamiento complejo. Barcelona: Gedisa.

Neira Martínez, Angie, Fernando Teddy Reyes Reyes y Bernardo Esteban Riffo OCARES (2015). «Experiencia académica y estrategias de comprensión lectora en estudiantes universitarios de primer año». Literatura y Lingüística, 31: 221-244. DOI: $10.4067 /$ So716-58112015000100012. 
Pacheco Peña, Eduardo y Sanyorei Porras Cose (2014). «Los momentos de la sesión a través de las rutas de aprendizaje: Propuesta de trabajo para los alumnos de las carreras de educación secundaria de la UNDAC». Horizonte de la Ciencia, 4 (7): 77-83. Disponible en http://bit.ly/2jqMxBJ.

Phan, Huy (2010). «Una perspectiva teórica del aprendizaje en el contexto del Pacífico: Una perspectiva sociocultural». Electronic Journal of Research in Educational Psychology, 8 (20): 411-428. Disponible en http://bit.ly/2ju7EU7.

Piaget, Jean (1968). Educación e instrucción. Buenos Aires: Proteo.

Pupo, Rigoberto (2004). Imagen, metáfora, verdad: Antología del pensamiento complejo. Tabasco: Universidad Popular de la Chontalpa.

-. (2014). Filosofía, educación cultura y pluralidad discursiva ensayística: Hacia una visión cultural y compleja del saber. Tepic: ISIC.

Reyes García, Freddy, Luis José Vera Guadrón y Elio Rafael Colina Caldera (2014). «Estrategias creativas para promover el aprendizaje significativo en la práctica docente simulada». Opción, 30 (75): 55-74. Disponible en http://bit.ly/2jrwXgf.

Ruiz Hoyos, Bayron Manuel y Dora Cardona Rivas (2016). «Propuesta de evaluación en el área básica del programa de medicina de la Universidad del Quindío 2008-2009 mediante la investigación-acción participativa». Revista Colombiana de Obstetricia y Ginecología, 67 (2): 89-100. DOI: 10.18597/rcog.378.

Sancho-Asensio, Andreu, Xavi Solé, José Montero, Joan Navarro, Xavi CaNALETA y David Vernet (2014). «Herramienta de soporte para la formación de grupos de trabajo en entornos de aprendizaje colaborativo». Iberian Conference on Information Systems and Technologies, CISTI 6876942. DOI: 10.1109/ CISTI.2014.6876942.

TAcca, María Carmen Villela Rosa y Ángela Uchoa Branco (2008). «Processos de significação na relação professor-alunos: Uma perspectiva sociocultural construtivista».Estudos de Psicologia, 13 (1):39-48.DOI:10.159o/S1413-294X2008000100005.

VAlDERramas Gomes, Cláudia Aparecida (2014). «A relação sujeito-objeto e a unidade afetivo-cognitiva: contribuições para a psicologia e para a educação». Psicologia Escolar e Educacional, 18 (1): 161-168. DOI: 10.1590/S1413-85572014000100017.

Vera-Villarroel, Pablo, Sebastián Lillo, Andrea Slachevsky y Jaime Silva (2010). «Emociones y neurociencia: Una aproximación desde la productividad científica».Revista Ecuatoriana de Neurología, 19 (1-2): 81-86. Disponible en http:// bit.ly/2jqoJeq.

Vigotsky, Lev (1979). El desarrollo de los procesos psíquicos superiores. Barcelona: Crítica.

-. (1987). Historia del desarrollo de las funciones psíquicas superiores. La Habana: Editorial Científico Técnica.

-. (1998). Pensamiento y lenguaje. Buenos Aires: Fausto.

-. (2004). Teoría de las emociones. Estudio histórico-psicológico. Madrid: Akal. 
Vidal-Abarca, Eduardo y Gabriel Martínez (1998). «Por qué los textos son difíciles de comprender? Las inferencias son la respuesta». Textos de Didáctica de la Lengua y de la Literatura, 16: 85-97.

Wenger, Etienne (2001). Comunidades de práctica, Aprendizaje, significado e identidad. Barcelona: Paidós Ibérica.

ZAPOROZHETS, Alexander Vladimirovich (1980). «La importancia de las primeras etapas de la niñez en la formación de la personalidad infantil». Infancia y Aprendizaje, 3 (11): 69-75. DOI: 10.1080/02103702.1980.10821808.

\section{Sobre los autores}

Taeli Gómez Francisco es abogada, académica asociada de la Cátedra de Filosofía del Derecho y Ética, Facultad de Ciencias Jurídicas y Sociales de la Universidad de Atacama, Chile. Doctora en Ciencias Filosóficas de La Universidad de La Habana, Cuba. Creadora y directora del Programa para la Enseñanza Integral del Derecho (PEID) del Departamento de Ciencias Jurídicas de la de la Universidad de Atacama. Sus correos electrónicos son taeli.gomez@uda.cl y taelig@yahoo.es.

JuAn Rubio González es psicólogo, integrante del Programa para la Enseñanza Integral del Derecho del Departamento de Ciencias Jurídicas de la de la Universidad de Atacama. Licenciado en Psicología y diplomado en Psicología Educativa y en Pensamiento Complejo en la Multiversidad Mundo Real Edgar Morin, México. Sus correos electrónicos son juan.rubio@uda.cly jj.rubio.72@gmail.com. 


\title{
REVISTA PEDAGOGÍA UNIVERSITARIA Y DIDÁCTICA DEL DERECHO
}

La Revista Pedagogía Universitaria y Didáctica del Derecho (RPUDD) es una publicación científica semestral que contribuye tanto a la reflexión multidisciplinaria sobre Pedagogía Universitaria y Didáctica del Derecho, para la formación y consolidación de esta área de investigación, como a la difusión de prácticas innovadoras en la enseñanza-aprendizaje del Derecho, considerando el contexto nacional e internacional..

\author{
DIRECTORA \\ María Francisca Elgueta Rosas \\ ASISTENTE EDITORIAL \\ Priscilla Saavedra Medina \\ SITIO WEB \\ pedagogiaderecho.uchile.cl \\ E-MAIL \\ rpedagogia@derecho.uchile.cl \\ LICENCIA DE ESTE ARTÍ́CULO
}

Creative Commons Atribución Compartir Igual 4.0 Internacional

\author{
9 \\ La edición de textos, el diseño editorial \\ y la conversión a formatos electrónicos de este artículo \\ han estado a cargo de Tipográfica \\ (www.tipografica.cl).
}

